\title{
The Show Must Go On: Using Proximie to Facilitate Collaboration between Experts to Perform a Complex Mitral Clip Procedure during COVID-19 \\ F Sawaya $^{1}$, A Zgheib ${ }^{1}$, JS Karim ${ }^{2 *}$ and N Hachach-Haram ${ }^{3}$
}

${ }^{1}$ Division of Cardiology, Interventional and Structural Cardiology, American University of Beirut Medical Center, Lebanon ${ }^{2}$ Proximie, The Harley Building, London, United Kingdom

${ }^{3}$ Guy's and St. Thomas' NHS Foundation Trust, King's Health Partners, United Kingdom

*Corresponding author: Jamila S Karim, Proximie, The Harley Building, 77 New Cavendish St., London, United Kingdom

\section{Introduction}

As COVID-19 spreads across the globe at an alarming pace, clinicians of all specialties are being forced to re-evaluate how they can continue to deliver the best quality of care to their patients during this turbulent time.

Although elective procedures have been canceled indefinitely in many countries, urgent interventional cardiology procedures are still being performed [1]. The interventional suite during COVID-19, however, is almost unrecognizable. Interventional suites used to be a welcome setting for trainees of all grades to watch and learn new procedures. Now, access is restricted to the minimum number of required clinical team members. Medical device experts were previously an invaluable source of support and knowledge where new or complex devices were being employed during a procedure. Current travel restrictions and infection control policies now impede their ability to provide direct technical support during procedures.

Paradoxically, measures that are in place to safeguard patients and clinicians against transmission of infection are also limiting the ability of experts to collaborate and share knowledge at a time when only the most critical, and often the most challenging, procedures are being performed on the most acutely unwell patients.
We present the case of a 79-year-old patient who required an urgent mitral clip for treatment of recurring acute decompensated pulmonary edema as a result of severe mitral regurgitation. An augmented reality (AR) platform, Proximie, was used to virtually connect an interventional cardiologist based at the American University of Beirut Medical Center (AUBMC) with a medical device expert in Europe intraoperatively in order to successfully perform the life-saving procedure using a novel medical device.

\section{The Proximie System}

Proximie is an augmented reality platform that enables clinicians to connect in real-time in order to share expertise and provide mentorship [2].

A live video feed of the procedure is captured using a camera, a capture card and a laptop or tablet. A remote expert using a laptop or tablet with a Chrome browser can access the feed in real-time via the online Proximie platform (Figure 1). The technology is end-toend encrypted for security and fully HIPAA and GDPR compliant. Proximie's augmented reality toolbox can be employed by the remote user to annotate the live feed or virtually reach into the field to indicate structures or provide precise guidance in real-time. Crucially, these tools enable a remote mentor to provide mentorship both by voice and by demonstration.

Citation: Sawaya F, Zgheib A, Karim JS, Hachach-Haram N (2020) The Show Must Go On: Using Proximie to Facilitate Collaboration between Experts to Perform a Complex Mitral Clip Procedure during COVID-19. Int J Clin Cardiol 7:183. doi.org/10.23937/2378-2951/1410183

Accepted: June 10, 2020; Published: June 12, 2020

Copyright: (c) 2020 Sawaya F, et al. This is an open-access article distributed under the terms of the Creative Commons Attribution License, which permits unrestricted use, distribution, and reproduction in any medium, provided the original author and source are credited. 


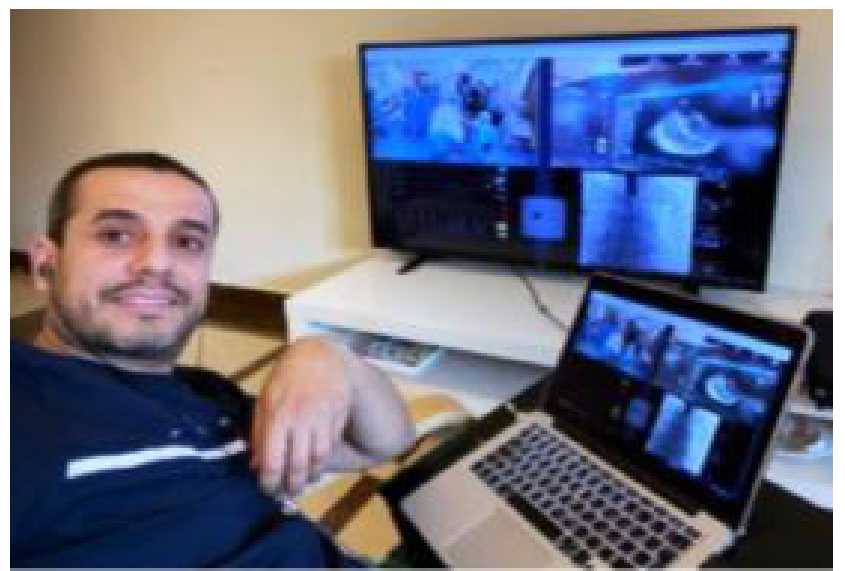

Figure 1: The Proximie platform being accessed by a remote user to view a live surgical procedure.

\section{Case Background}

\section{Case details}

A 79-year-old male patient was admitted to hospital in acute pulmonary edema, on the background of multiple recent hospital admissions for the same reason.

His extensive past medical history included coronary artery disease requiring coronary artery bypass graft surgery (CABG) 20 years prior, ischaemic cardiomyopathy (LVEF 20-24\%), chronic obstructive pulmonary disease (COPD) and atrial fibrillation.

He was transferred to a specialist center for further management and assessment for a possible mitral clip procedure.

The patient was tachypnoeic on arrival at the center. His heart rate and rhythm were irregular. An apical holosystolic murmur could be appreciated on auscultation. Diffuse crackles and bronchi were present across both lungs and there was decreased air entry bilaterally.

Pre-procedure trans-oesophageal echocardiography (TEE) demonstrated severely impaired LV systolic function with LVEF estimated at $20-24 \%$ and severe mitral valve regurgitation (Video 1 and Video 2).

The patient was optimised on admission. Levophed and dobutamine were started for treatment of the cardiogenic shock.

Unfortunately, the patient decompensated overnight and required intubation. The mitral clip procedure was performed the following day.

\section{Setup}

The procedure was performed at the AUBMC by a consultant interventional cardiologist and his fellow. Proximie was setup in the interventional suite pre-operatively. A remote medical device expert based in Europe accessed a live feed of the procedure using a standard laptop in order to provide guidance at key stages of the mitral clip deployment. He had access to the live fluoroscopy screen, the TEE screen and 2 cameras set-up

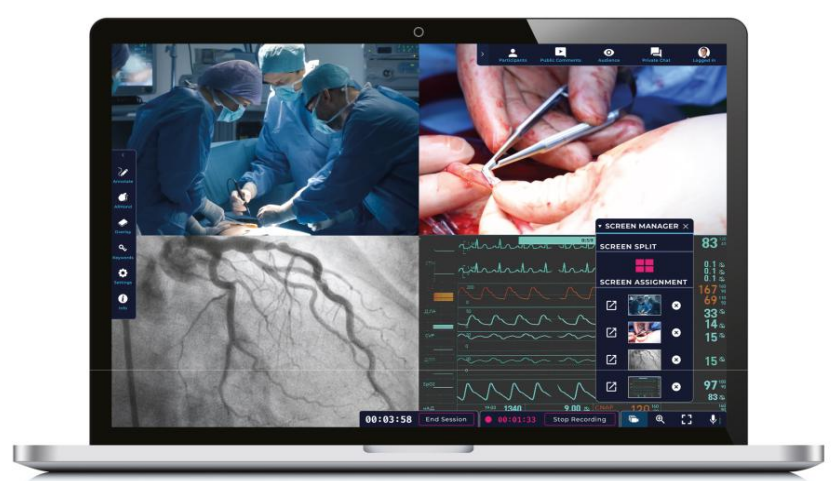

Figure 2: Remote four-camera view using Proximie.

strategically in the room with full voice control (Figure 2).

\section{Procedure}

The procedure was performed under general anesthesia. The right common femoral vein was punctured with a micropuncture needle and an $8 \mathrm{~F}$ sheath was introduced. An 8.5F SL-0 was introduced for transeptal puncture and positioned in the superior vena cava (SVC). Under TEE guidance, the septum was punctured with adequate height. After crossing the septum, an MP diagnostic catheter was positioned in the left atrium (LA) for continuous LA pressure monitoring. The delivery catheter was subsequently introduced into the LA over an amplatzer stiff wire.

Under 3D TEE guidance, a Mitral Clip NTR was delivered in a slightly medial position with reduction of mitral regurgitation from +4 to +1 (severe to mild) with a mean gradient of $2 \mathrm{mmHg}$ (Video 3, Video 4 and Video 5).

The groin was then closed with a figure of 8 stitch and manual compression was applied for $5 \mathrm{~min}$.

A TEE was performed intra-operatively. After the successful deployment of $1 \mathrm{clip}$ in the medial position, there was a significant reduction of MR from massive to mild regurgitation at an SBP of $160 \mathrm{mmHg}$ with normalization of flow reversal in the right pulmonary vein (Figure 3).

The patient remained haemodynamically stable following the procedure and was successfully extubated the following day.

\section{Discussion}

This case highlights the role that innovative technology solutions, such as the Proximie platform, can play in enabling interventional cardiology teams to continue to share expertise and perform ground-breaking, life-saving procedures during the COVID-19 pandemic.

The Proximie platform has historically been employed to facilitate global access to safe surgery and skill acquisition in order to reduce variation in care and improve patient outcomes. The lightweight platform is 


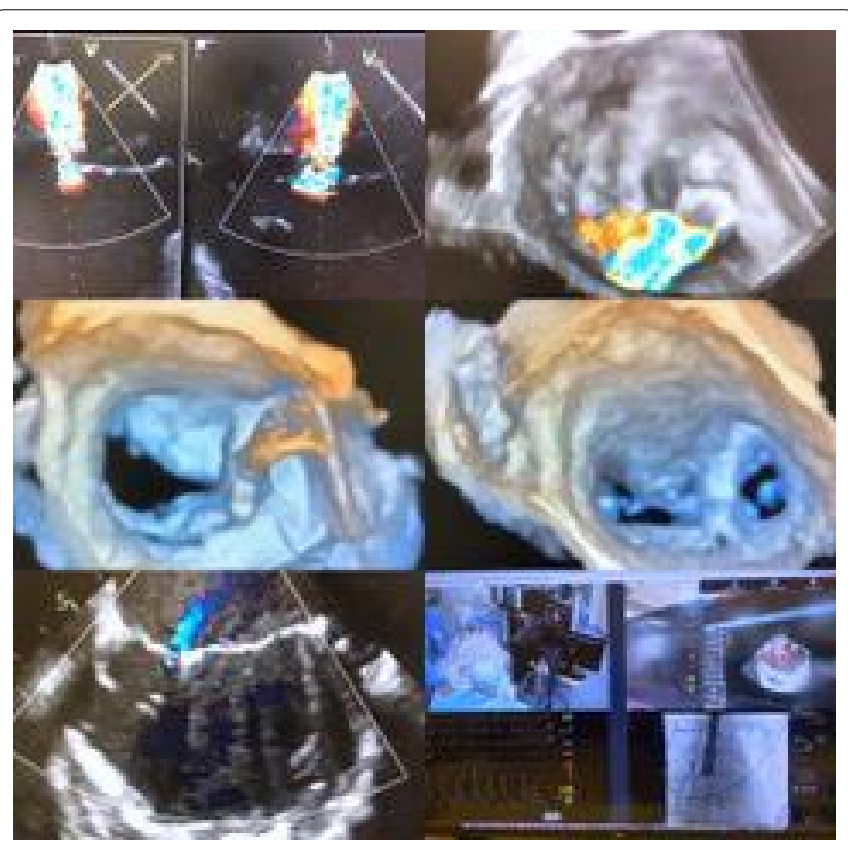

Figure 3: Trans-oesophageal echocardiogram.

intuitive, secure and easy to deploy in any setting. It has been tested in military environments and has successfully been used to connect surgeons in developing countries with international experts in their field [3].

At this critical time, interventional cardiologists face the near-impossible task of both safeguarding their patients and clinical teams against exposure to the highly infectious COVID-19 virus whilst also performing procedures on acutely unwell patients where input from multiple specialists or access to medical device experts may be required. Robust, tested technologies that enable remote collaboration can be used to overcome these challenges.

\section{Conclusion}

Current infection control measures and travel restrictions are impeding the ability of experts to collaborate. Patients undergoing complex procedures requiring input from multiple experts will be most affected by these constraints. Augmented reality tools, such as Proximie, can empower interventional cardiologists to share knowledge in real-time and provide optimum care while adhering to infection control and travel limitations.

\section{Conflict of Interest Declaration}

Dr. Jamila S Karim: Dr. Karim is the Head of Research at Proximie. This is a full-time paid position and she receives personal fees from Proximie. Proximie is an independent company that receives funding from personal investors and grants.

Dr. Nadine Hachach-Haram: Dr. Hachach-Haram is the Founder and CEO of Proximie.

\section{Authors Contribution}

Each listed author made substantial contributions to the writing of this case study.

\section{References}

1. Welt FGP, Shah PB, Aronow HD, Bortnick AE, Henry TD, et al. (2020) Catheterization laboratory considerations during the coronavirus (COVID-19) pandemic: From the ACC's Interventional Council and SCAI. J Am Coll Cardiol 75: 23722375.

2. (2020) Proximie - Expanding Virtual Surgical Collaboration.

3. Greenfield MJ, Luck J, Billingsley ML, Heyes R, Smith OJ, et al. (2018) Demonstration of the effectiveness of augmented reality telesurgery in complex hand reconstruction in Gaza. Plast Reconstr Surg Glob Open 6: e1708. 